Environment Conservation Journal 20 (1\&2) 101-108 , 2019

ISSN 0972-3099 (Print) 2278-5124 (Online)

Abstracted and Indexed

\title{
Water quality monitoring of three Lentic water bodies of Ujjain District (M.P.) India
}

\author{
Jatwa P., ${ }^{1}$ Shrivastava S., ${ }^{2}$ Bhasin S. $^{3}$ and Shukla A. N. ${ }^{4}$
}

Received: 25.10 .2018

Revised: 28.01.2019

Accepted: 10.03.2019

\begin{abstract}
The present study aimed to calculate water quality index (WQI) in order to assess the suitability of water for domestic use and drinking purposes. Water quality index (WQI) is valuable and unique rating to depict the overall water quality status in a single term that is helpful for the selection of appropriate treatment technique to meet the concerned issues. Water quality analysis were carried out on surface samples collected from three sampling sites Sahebkhedi lake, Undasa wetland and Purushottam sagar of Ujjain Dist. for the month of May 2017 to June 2018. The analysis of different parameters namely - pH, Hardness, Calcium, Chlorides, Alkalinity, D.O., BOD were carried out as per standard methods, prescribed as in the APHA (2005). The present study concludes that these water bodies are not fit for drinking purpose and domestic use because it accounts for high anthropogenic activities, worship rituals and several thousands of pilgrims take hoy bath.
\end{abstract}

Key Words: BOD, DO, Eutrophic, Lentic waterbodies, Water Pollution, Water Quality Index.

\section{Introduction}

Water is a precious life supporting natural resource for which we have countless uses that are all important to our lives. Water pollution is a phenomenon that is characterized by the deterioration of its quality as a result of various human activities. In India only $12 \%$ of people get good drinking water (Kudesia, 1980). Inadequate management of water resources as directly or indirectly resulted in the degradation of hydrological environment (Karanth, 1989). Every living beings needs water. Man must always have an adequate supply of potable water for his various daily needs. Specifically, man needs water for drinking, cooking, bathing, washing, agricultural purpose, manufacturing and industrial purposes. Water quality assessment is required for the management of waterbodies. Controlling water pollution is urgent for ecological sustainability of water resources as well as for underlying economic reasons and human health (Bhutiani et al., 2018). The WQI is used to assess the quality of major water supply sources representing the level of pollution (Sari and wan, 2008). The use of WQI

\section{Author's Address}

School of Studies in Zoology and Biotechnology, Vikram University, Ujjain (M.P.), India.

E-mail.: pjatwa@gmail.com in the first instance was used by Horton (1965) and Brown et al. (1970). Since then, diverse methods for the calculation of WQI have been evolved till date. The water quality criteria, on the basis of WQI, have been prescribed as excellent, good, medium, bad and unsuitable for drinking or very bad (Chaturvedi and Bassin, 2010). The present investigations was carried out to calculate the Water Quality Index (WQI) in order to assess the suitability of water collected from different three sampling sites in Ujjain (M.P.).

\section{Materials and Methods \\ Study Area}

Site I (Sahebkhedi Lake): It is situated near RD Gardi medical college on Agar road Ujjain (M.P.). This study site is situated at a distance of about 7 $\mathrm{km}$ from the Ujjain, its live capacity is $10.32 \mathrm{mq}$ and it has a dead storage is $2.15 \mathrm{mq}$., its length and water speed area are $3 \mathrm{~km}$ and 383 hectare respectively. In this lake used for fish culture, Irrigation and Public water supply.

Site II (Undasa wetland): Undasa wetland is located just outside the municipal limits of Ujjain city in M.P. This is situated at $75^{\circ} 50^{\prime}$ and $23^{\circ} 13^{\prime}$ on Ujjain- Maksi road along the Pingleshwar nala. The catchment area of this wetland extends up to 
$11.25 \mathrm{sq}$ miles with an average storage capacity of 186.668 mcft. Its live capacity is $5.32 \mathrm{mq}$., and its dead storage capacity is $0.09 \mathrm{mq}$. It has a water speed area 183.30 hectare and its length is $2.6 \mathrm{~km}$. It is used for drinking water supplies, irrigation and bathing. This is considered as a holy waterbody and several thousands of pilgrims take holy bath.

Site III (Purushottam sagar) : It is situated near Ankpat Marg, near Idgah of Ujjain City (M.P.) It is one of the important Pilgrims point of Ujjain also known as Solah sagar. Purushottam sagar is Perennial aquatic body, The average length and width are approximately 400 and 230 meters respectively. It is used for fish culture \& Trapa cultivation.

The present study has been conducted for a period of one year from July 2017 to June 2018. The Physico-chemical study includes a regular record of air temp., water temp., colour, odour, taste, turbidity, depth range, transparency, $\mathrm{pH}$, dissolved oxygen, $\mathrm{BOD}, \mathrm{COD}, \mathrm{CO}_{3}, \mathrm{HCO}_{3}$, total hardness, magnesium, calcium and chloride, The methods being used for the analysis of various physicochemical parameters are as given in standard method for the examination of water and waste water (APHA, 2005).

\section{Water Quality Index:}

WQI is a statistical way of calculating the pollution status of any water body. It was calculated by using formulae of (Brown et al., 1972).

$$
W Q I_{A}=\sum_{i=1}^{n} \text { wiqi } / \Sigma^{n}{ }_{i=1} w i
$$

Table 1 Water Quality Index (WQI) and status of water quality (Brown et al., 1972, Chatterji and Raziuddin, 2002).

\begin{tabular}{|l|l|}
\hline $\begin{array}{l}\text { Water Quality Index } \\
\text { Level }\end{array}$ & Water Quality Status \\
\hline $0-25$ & Excellent water quality \\
\hline $26-50$ & Good water quality \\
\hline $51-75$ & Poor water quality \\
\hline $76-100$ & Very Poor water quality \\
\hline Above 100 & Unsuitable for drinking \\
\hline
\end{tabular}

\section{Results and Discussion}

Growth, production, survival and reproduction of The results of the physicochemical analysis of the water samples collected and analysed for Sahebkhedi lake, Undasa wetland and Purushottam sagar are shown in tables $1,2,3$ while the statistics and calculated water quality index (WQI) shown in (Fig -1). The WQI has been used to integrate various water quality parameters and their aspect into a single frame showing a clear image of water quality of three water bodies Sahebkhedi lake, Undasa wetland and Purushottam sagar. The variation in WQI from July 2017 to June 2018 has been noticed (Fig. 1).

It is observed that higher values of WQI during summer season because of high pollution load and highly stressed condition followed by rainy and winter season. Yogendra and Puttaiah (2008), also observed that the pollution load is relatively high during summer season when compared to the winter and rainy season from urban waterbody in Shimoga Town, Karnataka. Minimum value of WQI is noticed in the month of December at Sahebkhedi lake study site and maximum were reported at Purushottam sagar in the month of June. This water quality rating study clearly shows that the status of the water is unsuitable for drinking purpose. Water quality index is reported in the order as Purushottam sagar > Undasa wetland > Sahebkhedi lake (Fig. 1). However, very high values of WQI are recorded from Purushottam sagar throughout the entire study period which indicates water bodies to be in highly polluted. This waterbody is unfit for drinking purpose and domestic use without proper treatment. The rapid increase of the day to day activities are considered as domestic wastes, the animal, fruits or vegetables residues (also called garbage). Worship materials like flowers, coconut etc and common waste products include nitrogenous compounds like Urea and ammomia and during rainy season gutter or dirt from the nearest colonies have been created pollution in the Pond and during festivals Jhankis and Dolgyaras immersion are also created pollution in this pond. Bhasin et al. (2016), also observed in similar variable physico-chemical values in Kshipra river. Undasa wetland is also polluted, and this water body is not suitable for drinking purpose because it accounts for high anthropogenic activities, soil erosion, land degradation, worship materials like flowers, coconut ets. from the temple and several thousands of pilgrims take holy bath. Jatwa et al. (2018), from Undasa wetland observed that Undasa wetland receives a considerable 
Water quality monitoring of three Lentic water bodies of U.j.jain District

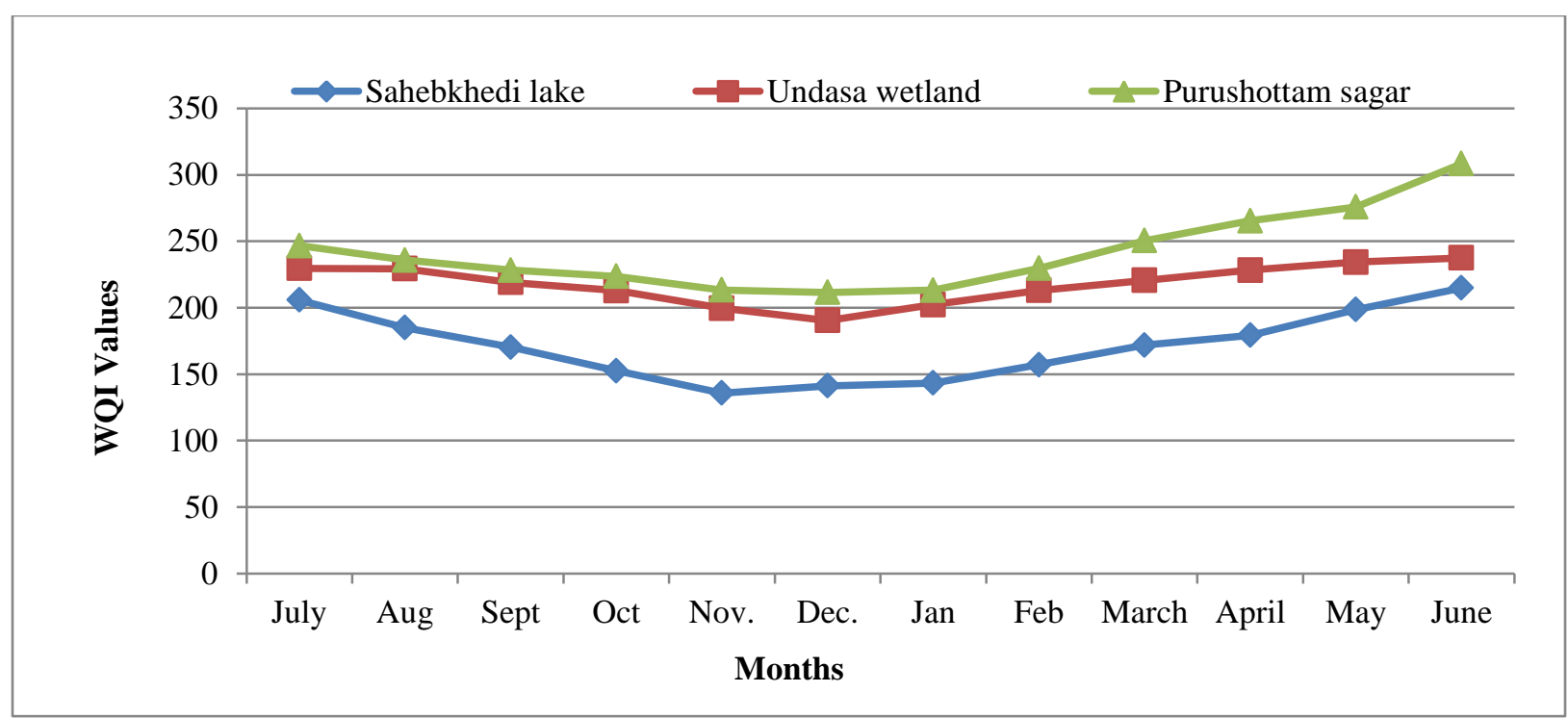

Fig 1. Water quality monitoring of three waterbodies of Ujjain District (M.P.)

amount of domestic water waste and sewage from nearby urban and rural areas. Sahebkhedi lake is moderate polluted, but this waterbody is unfit for drinking purpose without proper treatment. Yogendra and Puttaiah (2008), recorded Water Quality Index in different seasons indicate that poor quality of water in Urban waterbody in Shimoga Town, Karnataka.

Among all the physicochemical parameters selected for the water quality index calculations, $\mathrm{pH}$ is an important parameter which determines the suitability of water for various purposes. In the present study the values of $\mathrm{pH}$ fluctuated between 7.7- 8.4 at Sahebkhedi lake. The minimum value of pH 7.7 has been recorded during the winter 2017 however maximum value of $\mathrm{pH}$ has been observed during monsoon 2017 (Table-1). At Undasa wetland in $\mathrm{pH}$ values fluctuated between 7.9-8.6. The minimum value of $\mathrm{pH} 7.9$ has been recorded during the winter 2017 and maximum value of $\mathrm{pH}$ has been recorded during monsoon 2017 (Table-2). Similarly at Purushottam sagar in $\mathrm{pH}$ values fluctuated between 8.1-8.5. The minimum value of $\mathrm{pH} 8.1$ has been recorded during the winter season 2018 and maximum value of $\mathrm{pH}$ has been recorded during monsoon season 2017 (Table-3). Similar pattern was observed by Bhtuiani et al. (2018). In India many small confined water pockets are particularly alkaline in nature (Sharma et al., 1984). Total hardness is an important limnological parameter indicating level of water quality and organic production in the lake (Wetzel, 1983). The hardness values ranged between 132-164 mg/lt at Sahebkhedi lake (Table-1) followed by at Undasa wetland and Purushottam sagar hardness values range from 148-200 mg/lt and 172-216 mg/lt respectively (Table-2) (Table-3). More or less similar trend was observed by Khanna et al. (2014) and Bhutiani et al. (2018).

Calcium values ranged between 48.09 to 61.72 at Sahebkhedi lake (Table-1) followed by at Undasa wetland calcium ranged from 52.90 to 68.13 (Table-2) and similarly at Purushottam sagar calcium ranged from 60.12 - $72.94 \mathrm{mg} / \mathrm{lt}$ respectively (Table -3 ). Chloride occurs in all types of natural waters. The high concentration of chloride is considered to be an indication of pollution due to high organic waste of animal origin. At Sahebkhedi lake Chloride values range from 41.99 to $61.99 \mathrm{mg} / \mathrm{lt}$ (Table-1). At Undasa wetland and Purushottam sagar Chloride values range from $39.99-83.99$ and $51.99-78.99 \mathrm{mg} / \mathrm{lt}$ respectively (Table-2) (Table-3). Alkalinity value less than $100 \mathrm{mg} / \mathrm{l}$ is desirable for domestic use. However, in large quantities it imparts bitter taste to water. In the present study at Sahebkhedi lake the total alkalinity of the water samples is found in the range 154- $212 \mathrm{mg} / \mathrm{lt}$ (Table-1). At Undasa wetland and Purushottam sagar total alkalinity range from 170-242 and 198-268 mg/lt respectively (Table-2 and 3). More or less similar trend was observed by Bhutiani et al. (2018). 
Jatwa et al.

Table 1 : Monthly fluctuation of Physico - chemical parameters at Sahebkhedi Lake from July 2017- June 2018

\begin{tabular}{|c|c|c|c|c|c|c|c|c|c|c|c|c|c|}
\hline $\mathbf{S} \mathbf{N}$ & Parameter & July & Aug. & Sept & Oct. & Nov. & Dec. & Jan. & Feb & Mar & Apr. & May & June \\
\hline & Time & $\begin{array}{l}6: 40 \\
\mathrm{AM}\end{array}$ & $\begin{array}{l}7: 20 \\
\text { AM }\end{array}$ & $\begin{array}{l}7: 25 \\
\text { AM }\end{array}$ & $\begin{array}{l}8: 15 \\
\text { AM }\end{array}$ & $\begin{array}{l}6: 56 \\
\text { AM }\end{array}$ & $\begin{array}{l}8: 20 \\
\text { AM }\end{array}$ & $\begin{array}{l}6: 45 \\
\mathrm{AM} \\
\end{array}$ & $\begin{array}{l}7: 30 \\
\mathrm{AM}\end{array}$ & $\begin{array}{l}7: 30 \\
\mathrm{AM} \\
\end{array}$ & $\begin{array}{l}7: 15 \\
\text { AM }\end{array}$ & $\begin{array}{l}7: 10 \\
\mathrm{AM} \\
\end{array}$ & $\begin{array}{l}6: 43 \\
\mathrm{AM}\end{array}$ \\
\hline 1. & Env. Condition & $\begin{array}{l}\text { Sunny } \\
\text { day }\end{array}$ & $\begin{array}{l}\text { Cloudy } \\
\text { weather }\end{array}$ & $\begin{array}{l}\text { Cloudy } \\
\text { weather }\end{array}$ & $\begin{array}{l}\text { Cloudy } \\
\text { weather }\end{array}$ & $\begin{array}{l}\text { Cold } \\
\text { weather }\end{array}$ & $\begin{array}{l}\text { Cold } \\
\text { weather }\end{array}$ & $\begin{array}{l}\text { Cold } \\
\text { weather }\end{array}$ & $\begin{array}{l}\text { Cold } \\
\text { weather }\end{array}$ & $\begin{array}{l}\text { Clear } \\
\text { weather }\end{array}$ & $\begin{array}{l}\text { Sunny } \\
\text { weather }\end{array}$ & $\begin{array}{l}\text { Sunny } \\
\text { day }\end{array}$ & $\begin{array}{l}\text { Cloudy } \\
\text { weather }\end{array}$ \\
\hline 2. & Depth (m) & & & 3.41 & 3.41 & 3.34 & & 1.79 & & 0.43 & 0.6 & 0.73 & \\
\hline 3. & Air temp. ${ }^{\circ} \mathrm{C}$ & 21 & 21 & 17 & 22 & 19 & 14 & 10 & 13 & 15 & 26 & 27 & 25 \\
\hline 4. & Water temp. ${ }^{\circ} \mathrm{C}$ & 22 & 20 & 21 & 20 & 17 & 19 & 17 & 20 & 23 & 25 & 28 & 28 \\
\hline 5. & Water Colour & Muddy & Muddy & Muddy & Muddy & C.L. & C.L. & C.L. & C.L. & C.L. & C.L. & Greenish & Greenish \\
\hline 6. & Odour & O..L & O.L. & O.L. & O.L. & O.L. & O.L. & O.L. & O.L. & O.L. & O.L. & O.L. & O.L. \\
\hline 7. & Taste & T.L. & T.L. & T.L. & T.L. & T.L. & T.L. & T.L. & T.L. & T.L. & T.L. & T.L. & T.L. \\
\hline 8. & Turbidity (NTU) & 55 & 59 & 50 & 38 & 28 & 25 & 29 & 34 & 38 & 42 & 45 & 48 \\
\hline 9. & Transparency $(\mathrm{cm})$ & 50 & 38 & 60 & 98 & 124 & 129 & 109 & 76.2 & 74 & 68 & 60 & 56 \\
\hline 10. & $\mathrm{pH}$ & 8.1 & 8.4 & 8.2 & 8.0 & 7.9 & 7.7 & 7.7 & 7.50 & 7.8 & 7.9 & 8 & 8 \\
\hline 11. & $\begin{array}{l}\text { Dissolved Oxygen } \\
(\mathrm{mg} / \mathrm{l})\end{array}$ & 4.8 & 5.6 & 5.6 & 6 & 7.2 & 7.2 & 6.4 & 5.6 & 5.6 & 5.2 & 4.8 & 4.8 \\
\hline 12. & Carbonate $(\mathrm{mg} / \mathrm{l})$ & 14 & 14 & 12 & 12 & 10 & 10 & 8 & 10 & 12 & 12 & 12 & 16 \\
\hline 13. & Bicarbonate $(\mathrm{mg} / \mathrm{l})$ & 178 & 180 & 174 & 164 & 144 & 148 & 150 & 172 & 176 & 180 & 192 & 196 \\
\hline 14. & Total alkalinity $(\mathrm{mg} / \mathrm{l})$ & 192 & 194 & 186 & 176 & 154 & 158 & 158 & 182 & 188 & 192 & 204 & 212 \\
\hline 15. & Total hardness (mg/l) & 160 & 152 & 148 & 140 & 136 & 136 & 132 & 140 & 144 & 148 & 156 & 164 \\
\hline 16. & Calcium (mg/l) & 59.31 & 59.31 & 57.71 & 54.50 & 51.30 & 48.09 & 49.69 & 52.90 & 54.50 & 57.71 & 60.12 & 61.72 \\
\hline 17. & Magnesium (mg/l) & 2.891 & 0.947 & 0.940 & 0.950 & 1.924 & 3.862 & 1.919 & 1.915 & 1.916 & 0.940 & 1.422 & 2.394 \\
\hline 18. & Chloride (mg/l) & 59.99 & 55.99 & 53.99 & 53.99 & 49.99 & 45.99 & 41.99 & 43.99 & 46.99 & 51.99 & 55.99 & 61.99 \\
\hline 19. & $\mathrm{BOD}(\mathrm{mg} / \mathrm{l})$ & 20.2 & 17.2 & 15.6 & 13.8 & 12.4 & 12.8 & 13.4 & 15.2 & 16.6 & 17.2 & 19.4 & 21.6 \\
\hline 20. & $\mathrm{COD}(\mathrm{mg} / \mathrm{l})$ & 41.6 & 41.2 & 40.4 & 34.2 & 27.4 & 27.8 & 30.2 & 34.8 & 39.4 & 41.8 & 42.6 & 42.8 \\
\hline
\end{tabular}




\section{Water Quality Monitoring of three Lentic water bodies of Ujjain District}

Table 2 : Monthly fluctuation of Physico - chemical parameter at Undasa wetland from July 2017- June 2018

\begin{tabular}{|c|c|c|c|c|c|c|c|c|c|c|c|c|c|}
\hline $\mathbf{S ~ N}$ & Parameter & July & Aug. & Sept & Oct. & Nov. & Dec. & Jan. & Feb. & Mar. & Apri & May & June \\
\hline & Time & $\begin{array}{l}7: 35 \\
\text { AM }\end{array}$ & $\begin{array}{l}6: 38 \\
\text { AM }\end{array}$ & $\begin{array}{l}6: 50 \\
\mathrm{AM}\end{array}$ & $\begin{array}{l}7: 20 \\
\mathrm{AM}\end{array}$ & $\begin{array}{l}7: 44 \\
\mathrm{AM}\end{array}$ & $\begin{array}{l}7: 35 \\
\text { AM }\end{array}$ & $\begin{array}{l}6: 15 \\
\text { AM }\end{array}$ & $\begin{array}{l}7: 00 \\
\mathrm{AM}\end{array}$ & $\begin{array}{l}6: 55 \\
\mathrm{AM}\end{array}$ & $\begin{array}{l}6: 40 \\
\text { AM }\end{array}$ & $\begin{array}{l}6: 20 \\
\mathrm{AM}\end{array}$ & $\begin{array}{l}7: 20 \\
\mathrm{AM}\end{array}$ \\
\hline 1. & Env. Condition & $\begin{array}{l}\text { Partly } \\
\text { cloudy }\end{array}$ & $\begin{array}{l}\text { Cloudy } \\
\text { weather }\end{array}$ & $\begin{array}{l}\text { Cloudy } \\
\text { weather }\end{array}$ & $\begin{array}{l}\text { Cloudy } \\
\text { weather }\end{array}$ & $\begin{array}{l}\text { Cold } \\
\text { weather }\end{array}$ & $\begin{array}{l}\text { Cold } \\
\text { weather }\end{array}$ & $\begin{array}{l}\text { Cold } \\
\text { weather }\end{array}$ & $\begin{array}{l}\text { Cold } \\
\text { weather }\end{array}$ & $\begin{array}{l}\text { Clear } \\
\text { weather }\end{array}$ & $\begin{array}{l}\text { Sunny } \\
\text { day }\end{array}$ & $\begin{array}{l}\text { Clear } \\
\text { weather }\end{array}$ & $\begin{array}{l}\text { Sunny } \\
\text { Day }\end{array}$ \\
\hline 2. & Depth (m) & & & 2.23 & 2.25 & 1.95 & & 1.24 & & 0.69 & & 0.51 & \\
\hline 3. & Air temp. ${ }^{\circ} \mathrm{C}$ & 20 & 21 & 16 & 17 & 20 & 16 & 7 & 13 & 12 & 21 & 28 & 26 \\
\hline 4. & Water temp. ${ }^{\circ} \mathrm{C}$ & 21 & 20 & 20 & 19 & 17 & 19 & 16 & 20 & 22 & 25 & 29 & 28 \\
\hline 5. & Colour & Muddy & Muddy & Muddy & Muddy & C.L. & C.L. & C.L. & C.L. & C.L. & C.L. & Greenish & Greenish \\
\hline 6. & Odour & $\begin{array}{l}\text { Fishy } \\
\text { smell }\end{array}$ & $\begin{array}{l}\text { Fishy } \\
\text { smell }\end{array}$ & $\begin{array}{l}\text { Fishy } \\
\text { smell }\end{array}$ & $\begin{array}{l}\text { Fishy } \\
\text { smell }\end{array}$ & $\begin{array}{l}\text { Fishy } \\
\text { smell }\end{array}$ & O.L. & O.L. & O.L. & O.L. & O.L. & O.L. & O.L. \\
\hline 7. & Taste & T.L. & T.L. & T.L. & T.L. & T.L. & T.L. & T.L. & T.L. & T.L. & T.L. & T.L. & T.L. \\
\hline 8. & Turbidity (NTU) & 70 & 76 & 73 & 42 & 32 & 30 & 32 & 40 & 46 & 52 & 58 & 64 \\
\hline 9. & Transparency $(\mathrm{cm})$ & 43 & 30 & 28 & 81 & 120 & 122 & 114 & 70 & 68 & 64 & 55 & 52 \\
\hline 10. & $\mathrm{pH}$ & 8.4 & 8.6 & 8.4 & 8.2 & 8.2 & 8.0 & 7.9 & 8.0 & 8.1 & 8.2 & 8.2 & 8.3 \\
\hline 11. & $\begin{array}{l}\text { Dissolved Oxygen } \\
(\mathrm{mg} / \mathrm{l})\end{array}$ & 4.4 & 4.4 & 4.8 & 5.2 & 5.6 & 6.4 & 5.6 & 4.8 & 4.8 & 4 & 4.4 & 4.4 \\
\hline 12. & Carbonate (mg/l) & 16 & 16 & 14 & 12 & 12 & 10 & 12 & 14 & 12 & 14 & 16 & 18 \\
\hline 13. & Bicarbonate (mg/l) & 188 & 184 & 184 & 172 & 168 & 160 & 164 & 176 & 192 & 204 & 208 & 224 \\
\hline 14. & Total alkalinity $(\mathrm{mg} / \mathrm{l})$ & 204 & 200 & 198 & 184 & 180 & 170 & 176 & 190 & 204 & 218 & 224 & 242 \\
\hline 15. & Total hardness (mg/l) & 192 & 176 & 168 & 160 & 156 & 152 & 148 & 160 & 172 & 180 & 188 & 200 \\
\hline 16. & Calcium (mg/l) & 65.73 & 62.52 & 62.52 & 59.31 & 57.71 & 52.90 & 54.50 & 59.31 & 62.52 & 64.92 & 67.33 & 68.13 \\
\hline 17. & Magnesium (mg/l) & 6.764 & 4.833 & 2.889 & 2.891 & 2.892 & 4.839 & 2.888 & 2.891 & 3.853 & 4.339 & 4.821 & 7.259 \\
\hline 18. & Chloride (mg/l) & 78.99 & 77.99 & 77.99 & 73.99 & 69.99 & 39.99 & 47.99 & 55.99 & 57.99 & 63.99 & 81.99 & 83.99 \\
\hline 19. & BOD (mg/l) & 22.6 & 22.2 & 21.4 & 21.2 & 19.6 & 19.2 & 20.6 & 21.4 & 22.2 & 22.6 & 23.6 & 23.8 \\
\hline 20. & COD (mg/l) & 55.2 & 55.4 & 53.4 & 48.6 & 45.2 & 43.6 & 44.2 & 48.4 & 50.6 & 55.4 & 58.6 & 60.2 \\
\hline
\end{tabular}




\section{Jatwa et al.}

Table 3: Monthly fluctuation of Physico- chemical parameter at Purushottam Sagar from July 2017- June 2018

\begin{tabular}{|c|c|c|c|c|c|c|c|c|c|c|c|c|c|}
\hline $\mathbf{S ~ N}$ & Parameter & July & Aug. & Sept. & Oct. & Nov. & Dec. & Jan & Feb & Mar. & Apr. & May & June \\
\hline & Time & $\begin{array}{l}8: 20 \\
\text { AM }\end{array}$ & $\begin{array}{l}8: 10 \\
\text { AM }\end{array}$ & $\begin{array}{l}8: 8 \\
\text { AM }\end{array}$ & $\begin{array}{l}7: 50 \\
\text { AM }\end{array}$ & 6:56AM & $9: 25 \mathrm{AM}$ & $6: 30 \mathrm{AM}$ & 9:00AM & $\begin{array}{l}8: 38 \\
\text { AM }\end{array}$ & $\begin{array}{l}7: 00 \\
\text { AM }\end{array}$ & $\begin{array}{l}8: 18 \\
\text { AM }\end{array}$ & $\begin{array}{l}8: 4 \\
\mathrm{AM}\end{array}$ \\
\hline 1. & Env. Condition & $\begin{array}{l}\text { Sunny } \\
\text { day }\end{array}$ & $\begin{array}{l}\text { Cloud } \\
\mathrm{y}\end{array}$ & $\begin{array}{l}\text { Mostly } \\
\text { cloudy }\end{array}$ & $\begin{array}{l}\text { Partly } \\
\text { cloudy }\end{array}$ & $\begin{array}{l}\text { Cold } \\
\text { weather }\end{array}$ & $\begin{array}{l}\text { Cold } \\
\text { weather }\end{array}$ & $\begin{array}{l}\text { Winter } \\
\text { time }\end{array}$ & $\begin{array}{l}\text { Cold } \\
\text { weather }\end{array}$ & $\begin{array}{l}\text { Bright } \\
\text { sunny } \\
\text { day }\end{array}$ & $\begin{array}{l}\text { Sunny } \\
\text { Day }\end{array}$ & $\begin{array}{l}\text { Sunny } \\
\text { day }\end{array}$ & $\begin{array}{l}\text { Partly } \\
\text { cloudy }\end{array}$ \\
\hline 2. & Depth (m) & & 1.12 & & & & & 1.01 & & & 0.74 & 0.59 & 0.59 \\
\hline 3. & Air temp. ${ }^{\circ} \mathrm{C}$ & 20 & 19 & 18 & 23 & 20 & 15 & 7 & 17 & 26 & 23 & 25 & 25 \\
\hline 4. & Water temp. ${ }^{\circ} \mathrm{C}$ & 22 & 21 & 20 & 20 & 18 & 19 & 16 & 20 & 22 & 27 & 29 & 29 \\
\hline 5. & Water colour & Muddy & Muddy & Muddy & Muddy & $\begin{array}{l}\text { Greenis } \\
\mathrm{h}\end{array}$ & $\begin{array}{l}\text { Greenis } \\
\mathrm{h}\end{array}$ & $\begin{array}{l}\text { Greenis } \\
\mathrm{h}\end{array}$ & $\begin{array}{l}\text { Greenis } \\
\mathrm{h}\end{array}$ & $\begin{array}{l}\text { Greenis } \\
\mathrm{h}\end{array}$ & $\begin{array}{l}\text { Greenis } \\
\mathrm{h}\end{array}$ & Greenish & Greenish \\
\hline 6. & Odour & $\begin{array}{l}\text { Fishy } \\
\text { smell }\end{array}$ & $\begin{array}{l}\text { Fishy } \\
\text { smell }\end{array}$ & $\begin{array}{l}\text { Fishy } \\
\text { smell }\end{array}$ & $\begin{array}{l}\text { Fishy } \\
\text { smell }\end{array}$ & $\begin{array}{l}\text { Fishy } \\
\text { smell }\end{array}$ & $\begin{array}{l}\text { Fishy } \\
\text { smell }\end{array}$ & $\begin{array}{l}\text { Fishy } \\
\text { smell }\end{array}$ & $\begin{array}{l}\text { Fishy } \\
\text { smell }\end{array}$ & $\begin{array}{l}\text { Fishy } \\
\text { smell }\end{array}$ & $\begin{array}{l}\text { Fishy } \\
\text { smell }\end{array}$ & $\begin{array}{l}\text { Fishy } \\
\text { smell }\end{array}$ & $\begin{array}{l}\text { Fishy } \\
\text { smell }\end{array}$ \\
\hline 7. & Taste & Bitter & Bitter & Bitter & Bitter & Bitter & Bitter & Bitter & Bitter & Bitter & Bitter & Bitter & Bitter \\
\hline 8. & Turbidity (NTU) & 76 & 79 & 75 & 45 & 39 & 40 & 38 & 44 & 49 & 56 & 61 & 66 \\
\hline 9. & $\begin{array}{l}\text { Transparency } \\
(\mathrm{cm})\end{array}$ & 37 & 25 & 29 & 74 & 96 & 102 & 98 & 65 & 58 & 56 & 52 & 39 \\
\hline 10. & $\mathrm{pH}$ & 8.5 & 8.4 & 8.3 & 8.2 & 8.1 & 8.2 & 8.1 & 8.1 & 8.2 & 8.2 & 8.2 & 8.4 \\
\hline 11. & $\begin{array}{l}\text { Dissolved } \\
\text { Oxygen }(\mathrm{mg} / \mathrm{l})\end{array}$ & 4 & 4.8 & 4.8 & 5.2 & 5.2 & 5.6 & 6 & 4.8 & 4.4 & 4 & 3.6 & 3.2 \\
\hline 12. & Carbonate $(\mathrm{mg} / \mathrm{l})$ & 22 & 18 & 18 & 16 & 16 & 14 & 16 & 16 & 18 & 18 & 20 & 20 \\
\hline 13. & $\begin{array}{l}\text { Bicarbonate } \\
(\mathrm{mg} / \mathrm{l})\end{array}$ & 236 & 212 & 196 & 192 & 190 & 184 & 188 & 196 & 208 & 224 & 224 & 248 \\
\hline 14. & $\begin{array}{l}\text { Total alkalinity } \\
(\mathrm{mg} / \mathrm{l})\end{array}$ & 258 & 230 & 214 & 208 & 206 & 198 & 204 & 212 & 226 & 242 & 244 & 268 \\
\hline 15 . & $\begin{array}{l}\text { Total hardness } \\
(\mathrm{mg} / \mathrm{l})\end{array}$ & 208 & 204 & 200 & 192 & 180 & 172 & 180 & 184 & 188 & 196 & 204 & 216 \\
\hline 16. & Calcium (mg/l) & 72.14 & 68.13 & 66.53 & 64.12 & 62.52 & 60.12 & 62.52 & 64.92 & 66.53 & 68.13 & 70.54 & 72.94 \\
\hline 17. & $\begin{array}{l}\text { Magnesium } \\
(\mathrm{mg} / \mathrm{l})\end{array}$ & 6.762 & 8.224 & 8.222 & 7.741 & 5.796 & 5.320 & 5.796 & 5.311 & 5.307 & 6.280 & 6.761 & 8.228 \\
\hline 18. & Chloride (mg/l) & 72.99 & 69.99 & 67.99 & 64.99 & 62.99 & 59.99 & 51.99 & 54.99 & 63.99 & 65.99 & 73.99 & 77.99 \\
\hline 19. & $\mathrm{BOD}(\mathrm{mg} / \mathrm{l})$ & 24.4 & 23.6 & 22.8 & 22.6 & 21.4 & 21.2 & 21.8 & 23.4 & 25.8 & 27.6 & 28.8 & 32.6 \\
\hline 20 & COD (mg/l) & 70.4 & 64.6 & 60.2 & 58.4 & 54.6 & 53.8 & 55.6 & 61.4 & 67.2 & 69.8 & 75.2 & 77.4 \\
\hline
\end{tabular}


Dissolved Oxygen is an important parameter which is essential to the metabolism of all aquatic organisms that posses aerobic respiration. At Sahebkhedi lake the present investigation indicated that the concentration of dissolved oxygen fluctuated between $4.4-7.2 \mathrm{mg} / \mathrm{lt}$ (Table-1). Seasonally, the concentration of dissolved oxygen was more during monsoon and least during summer. The observation is in conformity with the observations of Reddy et al. (1982), Ghosh and George (1989), Swarnalatha and Narasingarao (1983) and Venkateswarlu (1993). At Undasa wetland Dissolved oxygen values range from 4-6.4 $\mathrm{mg} / \mathrm{lt}$ (Table-2). Similarly at Purushottam sagar D.O. values range from 3.2 to $6 \mathrm{mg} . / \mathrm{l}$ (Table-3).

BOD is a parameter to assess the organic load in a waterbody. Many researchers have recorded higher BOD values in polluted water. At sahebkhedi lake BOD values ranged between 12.4 to $21.6 \mathrm{mg} / \mathrm{lt}$ (Table-1). It was high during summer, being in conformity with the observation of Catterjee (1992). At Undasa wetland BOD values ranged from 19.2-23.8 mg/lt (Table-2). and at Purushottam sagar BOD values ranged between $21.8-32.6 \mathrm{mg} / \mathrm{lt}$ (Table-3). Similar variable physico-chemical values are recorded also in Lakes and Rivers by Ochuko et al. (2014); Boah et al. (2015) and Thomas \& Abraham (2018), pointed out that the water quality in terms of physico-chemical parameters at Pamba River, Kerala is well within the limits and can be used for irrigation, bathing and domestic purposes without any treatment. But also recommended that, it is desirable to monitor water resourses regularly to prevent the outbreak of diseases. The present investigation which is helpful to determine the quality of water and the purpose of the investigation is to make the people aware about the contamination of this waterbodies.

\section{Conclusion}

The results from the present Investigation clearly pointed out that the water quality of the Purushottam sagar is highly polluted as compared to the Undasa wetland and Sahebkhedi lake is reported moderate pollution condition. Therefore, it can be concluded through this study was to calculate the water quality index (WQI) of three water bodies in order to assess its suitability for drinking purposes. The water quality index (WQI) is a clear indication that untreated water from the Sahebkhedi lake, Undasa wetland and Purushottam sagar is of unsuitable for drinking and must therefore be treated before use to avoid water related diseases. Water contamination by various human activities is another severe problem which had produced deteroriation in the lentic water bodies of Ujjain. Various anthropogenic activities like dumping of waste flower \& other sewage material has led to an increase in various pollution indicating physicochemical parameters like hardness, calcium, chloride etc. Local people \& government should be aware and should take required measure to reduce the amount of pollutant $\&$ should work to conserve these water bodies.

\section{Acknowledgement}

Authors are thankful to Prof. M.S. Parihar Prof. \& Head, S.S. in Zoology \& Biotechnology, Vikram University, and Ujjain (M.P.) for providing necessary research facilities

\section{References}

APHA, 2005. Standard Methods for the Examination of Water and Wastewater, 21 ${ }^{\text {st }}$ edn. American Public Health Association WWA, Washington, D.C.

Boah, D. K., Twum S. B. and Pelig- Ba Kennath B. 2015. Mathematical Computation of Water Quality Index of Vea Dam in Upper East Region of Ghana, Environmental Sciences, 3(1): 11-16.

Bhasin, S., Shukla, A. N, Shrivastava, S. and Mishra, U. 2016. Control chart Model for Assessment of water quality of a Tropical River Kshipra Ujjain, India, Haya : The Saudi J. Life Sci., 1(1): 51-64.

Bhutiani, R., Ahamad, F., Tyagi, V. and Ram, K. 2018. Evaluation of water quality of River Malin using water quality index (WQI) at Najibabad, Bijnor (UP) India. Environment Conservation Journal 19 (1\&2): 191-201.

Bhutiani, R., Ram, K., Tyagi, V., Ahamad, F. and Kaushik, P. 2018. Assessment of ground water quality of Laksar block in district Haridwar, Uttarakhand. Environment Conservation Journal 19 (3): 123-128.

Bhutiani, R., Khanna, D. R., Ahamad, F. and Tyagi, V. 2018. Assessment of water quality status of Malin River at Najibabad, Bijnor (UP), Water Biology:281-307.

Bhutiani, R., Khanna, D. R., Ahamad, F. and Tyagi, V. 2018. Physico-chemical characterization of river Ganga at Haridwar, Uttarakhand, Water Biology:143-159. 


\section{Jatwa et al.}

Brown, R. M., cleiland, N. J. Mc., Deiniger, R. A. and Connor, O' M. F. A. 1972. Water quality index - crossing the physical barrier (Jenkis, S H ed) Proc. Intl. Conf. on water poll. Res. Jerusalem, 6: 787-797.

Chatterjee, A. A. 1992. Water quality of Nandakanan lake India., J. Environ. Hlth. 34(4): 329-333.

Chaturvedi, M. K. and Bassin, J. K. 2010. Assessing the water quality index of water treatment plant and bore wells, in Delhi, India. Environ. monit. and assess. 163(1-4): 449453.

Chatterjee, C. and Raziuddin, M. 2002. Determination of Water Quality Index (WQI) of a degraded river in Asansol industrial area (West Bengal). Nature, Environ. and Poll. Tech., 1(2): 181-189.

Ghosh, A. and George, J. P. 1989. Studies on the abiotic factors and zooplankton in a polluted urban reservoir Hussain Sagar, Hyderabad: Impact on water quality and Embryonic Development of Fishes. Indian $\boldsymbol{J}$. Environ.Hlth., 31(1): 49-59.

Horton, R. K. 1965. An index number system for rating water quality . J Water Poll. Control Feder, 37(3) : 300-306.

Jatwa, P., Shrivastava, S., Bhasin, S. and Shukla A. N. 2018. Seasonal Variation in Zooplankton Diversity of Undasa Wetland, Ujjain (M.P.), Shrinkhla Ek Shodhparak Vaicharik Patrika, 6(2):

Khanna, D.R., Bhutiani R. Tyagi, V., and Ahamad, F. 2014. Impact of Sugar Mill Effluent on Physico-chemical Properties of Malin River at Najibabad, Bijnore. Indian. $J$. Sci. Res.Spl. Ed \& NSESIR, :5-10.

Ochuko, U., Theddeus, O., Asuma Oghenero, O. and Ezo John E. 2014. A Comparative Assessment of Water Quality Index (WQI) and Suitability of River Ase for Domestic Water Supply in Urban and Rural Communities in Southern Nigeria, International Journal of Humanities and Social Science, $4(1)$ :
Reddy, K. R., Sacco, P. D., Graetz, D. A., Campbel, K.L. and Sinclair., L. R. 1982. Water treatment by aquatic ecosystem : Nutrient removal by reservoirs and flooded fields. $\boldsymbol{J}$. Environmental Management, 6(3): 261-271.

Karanth, K. R. 1989. Hydrogoogly . Tata Mc. Graw Hill publishing company Ltd., New Delhi.

Kudesia, V. P. 1980. Water pollution, Pragathi Prakashan, Meerut, India: 1-12.

Sari, I. and Wan, M. W. O. 2008. Assessing the water quality index of Air Itam Dam, Penang, Malaysia ICERT: 601605.

Sharma, M. S. and Durve. V. S. 1984. Eutrophication of the lake Pichhola in Udaipur, Rjasthan. Poll. Res. 3(2): 39-44.

Swarnalatha, N. and Narasingrao, A. 1993. Ecological investigation of two lentic environments with reference to cyanobacteria and water pollution. Indian J. Microbial. Ecol., 3: 41-48.

Thomas, N. and Abraham N. P. 2018. Seasonal Variation in the Physico Chemical Parameters of Middle Region of Pamba River, Kerala, India. International Journal of Scientific \& Engineering Reasearch, 9(4):

Venkateswarlu, V. 1993. Ecological studies on the rivers of Andhra Pradesh with special reference to water quality and pollution. Proc. Indian. Acad. Sci. (Plant Sci). 96: 495508.

Wetzel, R. G. 1983. Limnology, second edition Saunders College Publishing House, U.S.A. 767.

World Health Organisation 2010. International Standard for Drinking Water Guidelines for Water Quality, Geneva.

Yogendra, K. and Puttaiah E. T. 2008. Determination of Water Quality Index and Suitability of an Urban Waterbody in Shimoga Town, Karnataka. Proceedings of Taal 2007 : The $12^{\text {th }}$ World Lake Conference : $342-346$ 